\title{
GLOBAL SPHERICALLY SYMMETRIC CLASSICAL SOLUTION TO COMPRESSIBLE NAVIER-STOKES EQUATIONS WITH LARGE INITIAL DATA AND VACUUM
}

\author{
SHIJIN DING*, HUANYAO WEN*†, LEI YAO ${ }^{\ddagger}$, AND CHANGJIANG ZHU
}

\begin{abstract}
In the paper, we obtain a result on the existence and uniqueness of global spherically symmetric classical solutions to the compressible isentropic Navier-Stokes equations with vacuum in a bounded domain or exterior domain $\Omega$ of $\mathbb{R}^{n}(n \geq 2)$. Here, the initial data could be large. Besides, the regularities of the solutions are better than those obtained in 4, 5, 6]. The analysis is based on some new mathematical techniques and some new useful energy estimates. This is an extensive work of 4, 5, 6], where the global radially symmetric strong solutions, the local classical solutions in 3D and the global classical solutions in 1D were obtained, respectively. This paper can be viewed as the first result on the existence of global classical solutions with large initial data and vacuum in higher dimension.
\end{abstract}

Key words. Compressible Navier-Stokes equations, vacuum, global classical solution.

AMS subject classifications. 76D05, 76N10, 35B65.

1. Introduction. In this paper, we consider the initial-boundary value problem of compressible isentropic Navier-Stokes equations in a bounded domain or exterior domain $\Omega$ of $\mathbb{R}^{n}(n \geq 2)$ :

$$
\left\{\begin{array}{l}
\rho_{t}+\nabla \cdot(\rho \mathbf{u})=0, \rho \geq 0 \\
(\rho \mathbf{u})_{t}+\nabla \cdot(\rho \mathbf{u} \otimes \mathbf{u})+\nabla P(\rho)=\mu \triangle \mathbf{u}+(\mu+\lambda) \nabla(\nabla \cdot \mathbf{u})+\rho \mathbf{f}
\end{array}\right.
$$

for $(\mathbf{x}, t) \in \Omega \times(0,+\infty)$, where

$$
\Omega=\{\mathbf{x}|a<| \mathbf{x} \mid<b\}, 0<a<b \leq \infty, \mathbf{f}(\mathbf{x}, t)=f(|\mathbf{x}|, t) \frac{\mathbf{x}}{|\mathbf{x}|},
$$

$\rho$ and $\mathbf{u}=\left(u_{1}, u_{2}, \cdots, u_{n}\right)$ denote the density and the velocity respectively; $P(\rho)=$ $K \rho^{\gamma}$, for some constants $\gamma>1$ and $K>0$, is the pressure function; $\mathbf{f}$ is the external force; the viscosity coefficients $\mu$ and $\lambda$ satisfy the natural physical restrictions: $\mu>0$ and $2 \mu+n \lambda \geq 0$.

We consider the initial condition:

$$
\left.(\rho, \mathbf{u})\right|_{t=0}=\left(\rho_{0}, \mathbf{u}_{0}\right) \text { in } \bar{\Omega},
$$

and the boundary condition:

$$
\mathbf{u}(\mathbf{x}, t) \rightarrow 0, \text { as }|\mathbf{x}| \rightarrow a \text { or } b, \text { for } t \geq 0,
$$

where

$$
\rho_{0}(\mathbf{x})=\rho_{0}(|\mathbf{x}|), \quad \mathbf{u}_{0}(\mathbf{x})=u_{0}(|\mathbf{x}|) \frac{\mathbf{x}}{|\mathbf{x}|} .
$$

*School of Mathematical Sciences, South China Normal University, Guangzhou 510631, P.R. China (Ding: dingsj@scnu.edu.cn).

${ }^{\dagger}$ Department of Mathematics, Central China Normal University, Wuhan 430079, P.R. China (Wen: huanyaowen@hotmail.com)

${ }^{\ddagger}$ Department of Mathematics, Northwest University, Xi'an 710127, P.R. China (Yao: yaolei1056@hotmail.com).

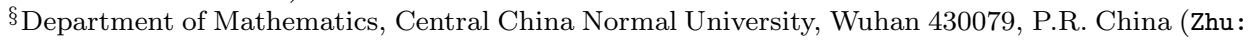
cjzhu@mail.ccnu.edu.cn) (Corresponding author). 
We are looking for a spherically symmetric classical solution $(\rho, \mathbf{u})$ :

$$
\rho(\mathbf{x}, t)=\rho(r, t), \mathbf{u}(\mathbf{x}, t)=u(r, t) \frac{\mathbf{x}}{r},
$$

where $r=|\mathbf{x}|$, and $(\rho, u)(r, t)$ satisfies

$$
\left\{\begin{array}{l}
\rho_{t}+(\rho u)_{r}+m \frac{\rho u}{r}=0, \rho \geq 0, \\
(\rho u)_{t}+\left(\rho u^{2}\right)_{r}+m \frac{\rho u^{2}}{r}+P_{r}=\nu\left(u_{r}+m \frac{u}{r}\right)_{r}+\rho f,
\end{array}\right.
$$

for $(r, t) \in(a, b) \times(0, \infty)$, with the initial condition:

$$
\left.(\rho(r, t), u(r, t))\right|_{t=0}=\left(\rho_{0}(r), u_{0}(r)\right) \text { in } I,
$$

and the boundary condition:

$$
u(r, t) \rightarrow 0, \text { as } r \rightarrow a \text { or } b, \text { for } t \geq 0,
$$

where $m=n-1, \nu=2 \mu+\lambda \geq \frac{2(n-1)}{n} \mu>0$ and $I=[a, b]$.

Let's review some previous work in this direction. When the viscosity coefficient $\mu$ is constant, the local classical solution of non-isentropic Navier-Stokes equations in Hölder spaces was obtained by Tani in [20] with $\rho_{0}$ being positive and essentially bounded. Using delicate energy methods in Sobolev spaces, Matsumura and Nishida showed in 17, 18, that the existence of the global classical solution provided that the initial data was small in some sense and away from vacuum. There are also some results about the existence of global strong solution to the Navier-Stokes equations with constant viscosity coefficient when $\rho_{0}>0$, refer for instance to [1, 15] for the isentropic flow. Jiang in 12 proved the global existence of spherically symmetric smooth solutions in Hölder spaces to the equations of a viscous polytropic ideal gas in the domain exterior to a ball in $\mathbb{R}^{n}(n=2$ or 3$)$ when $\rho_{0}>0$. For general initial data, Kawohl in [14] got the global classical solution with $\rho_{0}>0$ and the viscosity coefficient $\mu=\mu(\rho)$ satisfying

$$
0<\mu_{0} \leq \mu(\rho) \leq \mu_{1}, \text { for } \rho \geq 0,
$$

where $\mu_{0}$ and $\mu_{1}$ are constants. Indeed, such a condition includes the case $\mu(\rho) \equiv$ const.

In the presence of vacuum, Lions in [16] used the weak convergence method to show the existence of global weak solution to the Navier-Stokes equations for isentropic flow with general initial data and $\gamma \geq \frac{9}{5}$ in three dimensional space. Later, the restriction on $\gamma$ was relaxed by Feireisl, et al [9] to $\gamma>\frac{3}{2}$. Unfortunately, this assumption excludes for example the interesting case $\gamma=1.4$ (air, et al). Jiang and Zhang relaxed the condition to $\gamma>1$ in [13] when they considered the global spherically symmetric weak solution. It worths mentioning a result due to Hoff in [11, who showed the existence of weak solutions for the case $\gamma=1$ with $\rho_{0}$ being positive and essentially bounded.

There were few results about strong solution when the initial density may vanish until Salvi and Straškraba in [19], where $\Omega$ is a bounded domain, $P=P(\cdot) \in C^{2}[0, \infty)$, $\rho_{0} \in H^{2}, \mathbf{u}_{0} \in H_{0}^{1} \cap H^{2}$, and satisfied the compatibility condition:

$$
L \mathbf{u}_{0}(\mathbf{x})-\nabla P\left(\rho_{0}\right)(\mathbf{x})=\sqrt{\rho}_{0} \mathbf{g}, \text { for } \mathbf{g} \in L^{2},
$$

where $L:=\mu \Delta+(\mu+\lambda) \nabla$ div is the Lamé operator. 
Afterwards, Cho, Choe and Kim in [2, 3, 4, established some local and global existence results about strong solution in bounded or unbounded domain with initial data different from [19] still satisfying (1.7). Particularly, Choe and Kim in [4] showed the radially symmetric strong solution existed globally in time for $\gamma \geq 2$ in annular domain. As it is pointed out in 4 that the results have been proved only for annular domain and cannot be extended to a ball $\Omega=B_{R}=\left\{\mathbf{x} \in \mathbb{R}^{n}:|\mathbf{x}|<R<\infty\right\}$, because of a counter-example of Weigant [21]. Precisely, for $1<\gamma<1+\frac{1}{n-1}$, Weigant constructed a radially symmetric strong solution $(\rho, \mathbf{u})$ in $B_{R} \times[0,1)$ such that $\|\rho(\cdot, t)\|_{L^{\infty}\left(B_{R}\right)} \rightarrow \infty$ as $t \rightarrow 1^{-}$. A recent paper [8] written by Fan, Jiang and $\mathrm{Ni}$ improved the result in [4] to the case $\gamma \geq 1$.

The local classical solution was obtained by Cho and Kim in [5] when the initial density may vanish and satisfying the following compatibility conditions:

$$
L \mathbf{u}_{0}(\mathbf{x})-\nabla P\left(\rho_{0}\right)(\mathbf{x})=\rho_{0}\left[\mathbf{g}_{1}(\mathbf{x})-\mathbf{f}(\mathbf{x}, 0)\right],
$$

for $\mathbf{x} \in \bar{\Omega}, \mathbf{g}_{1} \in D_{0}^{1}$ and $\sqrt{\rho_{0}} \mathbf{g}_{1} \in L^{2}$. Recently, we used some new estimates to get a unique globally classical solution $\rho \in C^{1}\left([0, \infty) ; H^{3}\right)$ and $u \in H_{l o c}^{1}\left([0, \infty) ; H^{3}\right)$ to one dimensional compressible Navier-Stokes equations in a bounded domain when the initial density may vanish, cf. [6]. It worths mentioning that Xin in [22] showed that the smooth solution $(\rho, u) \in C^{1}\left([0, \infty) ; H^{3}\left(\mathbb{R}^{1}\right)\right)$ to the Cauchy problem must blow up when the initial density is of nontrivial compact support.

Since the system (1.4) have the one dimensional feature, the results in [6] are possible to be obtained here. Moreover, we get higher regularities of the solutions to system (1.4). This causes some new challenges compared with [6, which will be handled by some new estimates.

This paper can be viewed to be the first result on global classical solutions with large initial data and vacuum in higher dimension.

\section{Notations:}

(1) $Q_{T}=I \times[0, T], \widetilde{Q}_{T}=\bar{\Omega} \times[0, T]$ for $T>0$.

(2) For $p \geq 1, L^{p}=L^{p}(\Omega)$ denotes the $L^{p}$ space with the norm $\|\cdot\|_{L^{p}}$. For $k \geq 1$ and $p \geq 1, W^{k, p}=W^{k, p}(\Omega)$ denotes the Sobolev space, whose norm is denoted as $\|\cdot\|_{W^{k, p}} ; H^{k}=W^{k, 2}(\Omega)$.

(3) For an integer $k \geq 0$ and $0<\alpha<1$, let $C^{k+\alpha}(\bar{\Omega})$ denote the Schauder space of function on $\bar{\Omega}$, whose $k$ th order derivative is Hölder continuous with exponent $\alpha$, with the norm $\|\cdot\|_{C^{k+\alpha}}$.

(4) For an integer $k \geq 0$, denote

$$
H_{r}^{k}=H_{r}^{k}(I) \triangleq\left\{\left.u\left|\sum_{i=0}^{k} \int_{I} r^{m}\right| \partial_{r}^{i} u\right|^{2}<\infty\right\},
$$

with the norm

$$
\|\cdot\|_{H_{r}^{k}}=\left(\sum_{i=0}^{k} \int_{I} r^{m}\left|\partial_{r}^{i} u\right|^{2}\right)^{\frac{1}{2}}
$$

$L_{r}^{2}=H_{r}^{0}$.

(5) $D^{k, p}=\left\{v \in L_{l o c}^{1}(\Omega) \mid\left\|\nabla^{k} v\right\|_{L^{p}}<\infty\right\}, D^{k}=D^{k, 2}$.

(6) $D_{0}^{1}=D_{0}^{1,2}$ is the closure of $C_{0}^{\infty}(\Omega)$ in $D^{1,2}$. 
Our main results are stated as follows.

TheOrem 1.1. Assume that $\rho_{0} \geq 0$ satisfies $\rho_{0} \in L^{1} \cap H^{2}, \rho_{0}^{\gamma} \in H^{2}, \mathbf{u}_{0} \in D^{3} \cap D_{0}^{1}$ and $\mathbf{f} \in C\left([0, \infty) ; H^{1}\right), \mathbf{f}_{t} \in L_{l o c}^{2}\left([0, \infty) ; L^{2}\right)$, and the initial data $\rho_{0}, \mathbf{u}_{0}$ satisfy the compatible condition (1.8) with $\mathbf{g}_{1}(\mathbf{x})=g_{1}(r) \frac{\mathbf{x}}{r}$. Then for any $T>0$, there exists a unique global classical solution $(\rho, \mathbf{u})$ to (1.1)-(1.3) satisfying

$\left(\rho, \rho^{\gamma}\right) \in C\left([0, T] ; H^{2}\right), \rho \geq 0, \mathbf{u} \in C\left([0, T] ; D^{3} \cap D_{0}^{1}\right), \mathbf{u}_{t} \in L^{\infty}\left([0, T] ; D_{0}^{1}\right) \cap L^{2}\left([0, T] ; D^{2}\right)$.

REMARK 1.1. (i) Note that if $\Omega$ is bounded and locally Lipschitzian, then $D^{k, p}=$ $W^{k, p}$. See [10] for the proof.

(ii) By Sobolev embedding theorems, we have

$$
H^{k}(I) \hookrightarrow C^{k-\frac{1}{2}}(I), \text { for } k=1,2,3,
$$

this together with the regularities of $(\rho, u)$ give

$$
\left(\rho, \rho^{\gamma}\right) \in C\left([0, T] ; C^{1+\frac{1}{2}}(I)\right), u \in C\left([0, T] ; C^{2+\frac{1}{2}}(I)\right) .
$$

Since $(\rho(\mathbf{x}, t), \mathbf{u}(\mathbf{x}, t))=\left(\rho(r, t), u(r, t) \frac{\mathbf{x}}{r}\right)$, we get

$$
\left(\rho, \rho^{\gamma}\right) \in C\left([0, T] ; C^{1+\frac{1}{2}}(\bar{\Omega})\right), \mathbf{u} \in C\left([0, T] ; C^{2+\frac{1}{2}}(\bar{\Omega})\right),
$$

which means $(\rho, \mathbf{u})$ is the classical solution to (1.1)-(1.3).

TheOrem 1.2. Consider the same assumptions as in Theorem 1.1, and in addition assume that $\rho_{0} \in H^{5}, \rho_{0}^{\gamma} \in H^{5}, \nabla\left(\sqrt{\rho_{0}}\right) \in L^{\infty}, \sqrt{\rho_{0}} \nabla^{2} \mathbf{g}_{1} \in L^{2}, \rho_{0} \nabla^{3} \mathbf{g}_{1} \in L^{2}$, $\mathbf{u}_{0} \in D^{5}, \mathbf{f} \in C\left([0, \infty) ; H^{3}\right) \cap L_{l o c}^{2}\left([0, \infty) ; H^{4}\right), \mathbf{f}_{t} \in C\left([0, \infty) ; H^{1}\right) \cap L_{l o c}^{2}\left([0, \infty) ; H^{2}\right)$ and $\mathbf{f}_{t t} \in L_{l o c}^{2}\left([0, \infty) ; L^{2}\right)$. Then the regularities of the solution obtained in Theorem 1.1 can be improved as follows:

$$
\begin{gathered}
\left(\rho, \rho^{\gamma}\right) \in C\left([0, T] ; H^{5}\right), \mathbf{u} \in L^{\infty}\left([0, T] ; D_{0}^{1} \cap D^{5}\right) \cap L^{2}\left([0, T] ; D^{6}\right), \\
\mathbf{u}_{t} \in L^{\infty}\left([0, T] ; D_{0}^{1}\right) \cap L^{2}\left([0, T] ; D^{3}\right),\left(\sqrt{\rho} \nabla^{2} \mathbf{u}_{t}, \rho \nabla^{3} \mathbf{u}_{t}\right) \in L^{\infty}\left([0, T] ; L^{2}\right) .
\end{gathered}
$$

The rest of the paper is organized as follows. In Section 2, we prove Theorem 1.1. In Section 3, we prove Theorem 1.2 by giving some estimates similar to [6] and some new estimates, such as Lemma 3.6. Lemma 3.7 and Lemma 3.8.

The constants $\nu$ and $K$ play no role in the analysis, so we assume $\nu=K=1$ without loss of generality.

2. Proof of Theorem 1.1. In this section, we get a unique global classical solution to (1.4)-(1.6) with initial density $\rho_{0} \geq \delta>0$ and $b<\infty$ by some $a$ priori estimates globally in time based on the local solution. Moreover, the estimates are independent of $b$ and $\delta$. Next, we construct a sequence of approximate solutions to (1.4)-(1.6) under the assumption $\rho_{0} \geq \delta>0$. We obtain the global classical solution to (1.4)-(1.6) for $\rho_{0} \geq 0$ and $b<\infty$ after taking the limits $\delta \rightarrow 0$. Based on the global classical solution for the case of $b<\infty$, where the estimates are uniform for $b$, we can get the solution in the exterior domain by using similar arguments as in [3].

In the section, we denote by " $c$ " the generic constant depending on $a,\left\|\rho_{0}\right\|_{H^{2}}$, $\left\|\rho_{0}^{\gamma}\right\|_{H^{2}},\left\|\mathbf{u}_{0}\right\|_{D_{0}^{1}},\left\|\mathbf{u}_{0}\right\|_{D^{3}}, T$ and some other known constants but independent of $\delta$ and $b$.

Before proving Theorem 1.1, we give the following auxiliary theorem. 
TheOREm 2.1. Consider the same assumptions as in Theorem 1.1, and in addition assume that $\rho_{0} \geq \delta>0$ and $b<\infty$. Then for any $T>0$, there exists a unique global classical solution $(\rho, u)$ to $(1.4)$-(1.6) satisfying

$$
\begin{array}{cl}
\rho \in C\left([0, T] ; H^{2}(I)\right), & \rho \geq \frac{\delta}{c}, u_{t t} \in L^{2}\left([0, T] ; L^{2}(I)\right), \\
u \in C\left([0, T] ; H^{3}(I) \cap H_{0}^{1}(I)\right), & u_{t} \in C\left([0, T] ; H_{0}^{1}(I)\right) \cap L^{2}\left([0, T] ; H^{2}(I)\right) .
\end{array}
$$

The local solution in Theorem 2.1 can be obtained by the successive approximations as in [3, 5], we omit it here for simplicity. The regularities guarantee the uniqueness (refer for instance to [2, 3]). Based on it, Theorem 2.1 can be proved by some a priori estimates globally in time.

For $T \in(0,+\infty)$, let $(\rho, u)$ be the classical solution of (1.4)-(1.6) as in Theorem 2.1. Then we have the following estimates (cf. [4] and [8]):

Lemma 2.1. For any $0 \leq t \leq T$, it holds that

$$
\left\|\left(\rho, \rho^{\gamma}\right)\right\|_{H_{r}^{2}}+\left\|\left(\rho_{t},\left(\rho^{\gamma}\right)_{t}\right)\right\|_{H_{r}^{1}}+\int_{0}^{T}\left\|\left(\rho_{t t},\left(\rho^{\gamma}\right)_{t t}\right)\right\|_{L_{r}^{2}}^{2} \leq c, \rho \geq \frac{\delta}{c},
$$

and

$$
\int_{I}\left(r^{m} \rho u_{t}^{2}+r^{m} u_{r r}^{2}+r^{m} u_{r}^{2}+r^{m-2} u^{2}\right)+\int_{Q_{T}}\left(r^{m} u_{r t}^{2}+r^{m-2} u_{t}^{2}+r^{m} u_{r r r}^{2}\right) \leq c,
$$

where $\left\|\left(h_{1}, h_{2}\right)\right\|_{X}=\left\|h_{1}\right\|_{X}+\left\|h_{2}\right\|_{X}$, for some Banach space $X$, and $h_{i} \in X, i=1$,2.

REMARK 2.1. (i) For the estimates about $\rho^{\gamma}$, since $\rho$ and $\rho^{\gamma}$ satisfy the linear transport equations: $\rho_{t}+\mathbf{u} \cdot \nabla \rho+\rho \nabla \cdot \mathbf{u}=0$ and $\left(\rho^{\gamma}\right)_{t}+\mathbf{u} \cdot \nabla\left(\rho^{\gamma}\right)+\gamma \rho^{\gamma} \nabla \cdot \mathbf{u}=0$ respectively, then by using the similar arguments as that of Lemma 3.6 in [4], we get

$$
\begin{aligned}
& \frac{d}{d t}\left\{\int_{\Omega}\left|\nabla^{2} \rho\right|^{2}+\int_{\Omega}\left|\nabla^{2}\left(\rho^{\gamma}\right)\right|^{2}\right\} \\
\leq & c\|\nabla \mathbf{u}(\cdot, t)\|_{H^{1}}\left(\|\nabla \rho(\cdot, t)\|_{H^{1}}^{2}+\left\|\nabla\left(\rho^{\gamma}\right)(\cdot, t)\right\|_{H^{1}}^{2}\right) \\
& +c\left(\left\|\nabla^{2} G(\cdot, t)\right\|_{L^{2}}+\left\|\nabla^{2}\left(\rho^{\gamma}\right)(\cdot, t)\right\|_{L^{2}}\right)\left(\left\|\nabla^{2} \rho(\cdot, t)\right\|_{L^{2}}+\left\|\nabla^{2}\left(\rho^{\gamma}\right)(\cdot, t)\right\|_{L^{2}}\right) \\
\leq & c\left(\|\nabla \rho(\cdot, t)\|_{H^{1}}^{2}+\left\|\nabla\left(\rho^{\gamma}\right)(\cdot, t)\right\|_{H^{1}}^{2}+\|G(\cdot, t)\|_{H^{2}}^{2}\right),
\end{aligned}
$$

where $G=\nu \nabla \cdot \mathbf{u}-\rho^{\gamma}$ is the effective viscous flux; we have used the estimates $\|\mathbf{u}(\cdot, t)\|_{D_{0}^{1}} \leq c,\|\mathbf{u}(\cdot, t)\|_{D^{2}} \leq c$ and $\|\rho(\cdot, t)\|_{H^{1}} \leq c$ in [4]. Since $\|G(\cdot, t)\|_{H^{2}} \in$ $L^{2}(0, T)$ given in [4], hence, an application of the Gronwall inequality gives

$$
\left\|\left(\rho, \rho^{\gamma}\right)(\cdot, t)\right\|_{H^{2}} \leq c,
$$

where we have used the following Sobolev inequalities for radially symmetric functions defined in $\Omega^{\prime}=\left\{\mathbf{x} \in \mathbb{R}^{n} ;|x|>a>0\right\}:\|\nabla \rho\|_{L^{\infty}} \leq c\|\nabla \rho\|_{H^{1}},\|\nabla \mathbf{u}\|_{L^{\infty}} \leq c\|\nabla \mathbf{u}\|_{H^{1}}$. From $(*)$ and a direct calculation, we get

$$
\left\|\left(\rho, \rho^{\gamma}\right)\right\|_{H_{r}^{2}} \leq c .
$$

(ii) For the case $a \geq 0$ and $\gamma$ different from that in [21], it is interesting to investigate the existence of global spherically symmetric strong solutions or spherically symmetric classical solutions. We leave it as a forthcoming paper.

Lemma 2.2. For any $0 \leq t \leq T$, it holds

$$
\int_{I}\left(r^{m} u_{r t}^{2}+r^{m-2} u_{t}^{2}\right)+\int_{Q_{T}} r^{m} \rho u_{t t}^{2} \leq c .
$$


Proof. From (1.4), we get

$$
\rho u_{t}+\rho u u_{r}+\left(\rho^{\gamma}\right)_{r}=\left(u_{r}+\frac{m u}{r}\right)_{r}+\rho f .
$$

Differentiating (2.1) with respect to $t$, we have

$$
\rho u_{t t}+\rho_{t} u_{t}+\rho_{t} u u_{r}+\rho u_{t} u_{r}+\rho u u_{r t}+\left(\rho^{\gamma}\right)_{r t}=u_{r r t}+m r^{-2}\left(r u_{r t}-u_{t}\right)+\rho_{t} f+\rho f_{t} .
$$

Multiplying (2.2) by $r^{m} u_{t t}$, integrating over $I$, and using integration by parts, Lemma 2.1 and Cauchy inequality, we have

$$
\begin{aligned}
& \int_{I} r^{m} \rho u_{t t}^{2}+\frac{1}{2} \frac{d}{d t} \int_{I}\left(r^{m} u_{r t}^{2}+m r^{m-2} u_{t}^{2}\right) \\
= & \int_{I} r^{m}\left(\rho_{t} f+\rho f_{t}\right) u_{t t}-\int_{I} r^{m}\left[\rho_{t} u_{t}+\rho_{t} u u_{r}+\rho u_{t} u_{r}+\rho u u_{r t}+\left(\rho^{\gamma}\right)_{r t}\right] u_{t t} \\
\leq & -\int_{I} r^{m} \rho_{t} u_{t} u_{t t}-\int_{I} r^{m}\left(\rho_{t} u u_{r}-\rho_{t} f\right) u_{t t}+c \int_{I} r^{m} \rho u_{t}^{2}+\frac{1}{2} \int_{I} r^{m} \rho u_{t t}^{2}+c \int_{I} r^{m} u_{r t}^{2} \\
& -\int_{I} r^{m}\left(\rho^{\gamma}\right)_{r t} u_{t t}+c \int_{I} r^{m} f_{t}^{2},
\end{aligned}
$$

where we have used Lemma 2.1 and the following inequality found in [4:

$$
\sup _{a \leq r \leq b}|u(r, t)| \leq c\left[\int_{I}\left(r^{m} u_{r}^{2}+m r^{m-2} u^{2}\right)(r, t) d r\right]^{\frac{1}{2}}, \text { for } u(a, t)=0,
$$

and the one-dimensional Sobolev inequality:

$$
\sup _{a \leq r \leq b}|\varphi(r)| \leq c\|\varphi\|_{H^{1}(I)} .
$$

Thus

$$
\begin{aligned}
& \frac{1}{2} \int_{I} r^{m} \rho u_{t t}^{2}+\frac{1}{2} \frac{d}{d t} \int_{I}\left(r^{m} u_{r t}^{2}+m r^{m-2} u_{t}^{2}\right) \\
\leq & -\int_{I} r^{m} \rho_{t} u_{t} u_{t t}-\int_{I} r^{m}\left(\rho_{t} u u_{r}-\rho_{t} f\right) u_{t t}-\int_{I} r^{m}\left(\rho^{\gamma}\right)_{r t} u_{t t}+c \int_{I} r^{m} u_{r t}^{2}+c \int_{I} r^{m} f_{t}^{2}+c \\
= & -\frac{d}{d t} \int_{I}\left[\frac{1}{2} r^{m} \rho_{t} u_{t}^{2}+r^{m}\left(\rho_{t} u u_{r}-\rho_{t} f\right) u_{t}\right]+\frac{1}{2} \int_{I} r^{m} \rho_{t t} u_{t}^{2} \\
& +\int_{I} r^{m}\left(\rho_{t t} u u_{r}+\rho_{t} u_{t} u_{r}+\rho_{t} u u_{r t}-\rho_{t t} f-\rho_{t} f_{t}\right) u_{t}-\int_{I} r^{m}\left(\rho^{\gamma}\right)_{r t} u_{t t}+c \int_{I} r^{m} u_{r t}^{2} \\
& +c \int_{I} r^{m} f_{t}^{2}+c \\
\leq & -\frac{d}{d t} \int_{I}\left[\frac{1}{2} r^{m} \rho_{t} u_{t}^{2}+r^{m}\left(\rho_{t} u u_{r}-\rho_{t} f\right) u_{t}\right]-\frac{1}{2} \int_{I}\left(r^{m} \rho u\right)_{r t} u_{t}^{2}+c\left\|u_{t}\right\|_{L^{\infty}} \int_{I} r^{m}\left(\rho_{t t}^{2}+u^{2} u_{r}^{2}\right) \\
& +c\left\|u_{t}\right\|_{L^{\infty}}^{2} \int_{I} r^{m}\left(\rho_{t}^{2}+u_{r}^{2}\right)+c\left\|u_{t}\right\|_{L^{\infty}}\left(\int_{I} r^{m} \rho_{t}^{2}\right)^{\frac{1}{2}}\left(\int_{I} r^{m} u_{r t}^{2}\right)^{\frac{1}{2}} \\
& +\left\|u_{t}\right\|_{L^{\infty}} \int_{I} r^{m}\left(\rho_{t t}^{2}+f^{2}+\rho_{t}^{2}+f_{t}^{2}\right)-\int_{I} r^{m}\left(\rho^{\gamma}\right)_{r t} u_{t t}+c \int_{I} r^{m} u_{r t}^{2}+c \int_{I} r^{m} f_{t}^{2}+c,
\end{aligned}
$$


where we have used (1.4) $1,(2.3)$, Cauchy inequality, Hölder inequality and Lemma2.1. Using (2.3), Cauchy inequality, Lemma 2.1] again, along with (2.4) and integration by parts, we further obtain

$$
\begin{aligned}
& \frac{1}{2} \int_{I} r^{m} \rho u_{t t}^{2}+\frac{1}{2} \frac{d}{d t} \int_{I}\left(r^{m} u_{r t}^{2}+m r^{m-2} u_{t}^{2}\right) \\
\leq & -\frac{d}{d t} \int_{I}\left[\frac{1}{2} r^{m} \rho_{t} u_{t}^{2}+r^{m}\left(\rho_{t} u u_{r}-\rho_{t} f\right) u_{t}\right]+\int_{I} r^{m}\left(\rho u_{t}+\rho_{t} u\right) u_{t} u_{r t} \\
& +c\left[\int_{I}\left(r^{m} u_{r t}^{2}+m r^{m-2} u_{t}^{2}\right)\right]^{\frac{1}{2}}\left[\int_{I} r^{m}\left(\rho_{t t}^{2}+f^{2}+f_{t}^{2}\right)+1\right]+c \int_{I}\left(r^{m} u_{r t}^{2}+m r^{m-2} u_{t}^{2}\right) \\
& -\int_{I} r^{m}\left(\rho^{\gamma}\right)_{r t} u_{t t}+c \int_{I} r^{m} f_{t}^{2}+c \\
\leq & -\frac{d}{d t} \int_{I}\left[\frac{1}{2} r^{m} \rho_{t} u_{t}^{2}+r^{m}\left(\rho_{t} u u_{r}-\rho_{t} f\right) u_{t}\right]+c \int_{I} r^{m}\left(\rho^{2} u_{t}^{4}+\rho_{t}^{2} u^{2} u_{t}^{2}\right) \\
& +c \int_{I}\left(r^{m} u_{r t}^{2}+m r^{m-2} u_{t}^{2}\right)\left[\int_{I} r^{m}\left(\rho_{t t}^{2}+f_{t}^{2}\right)+1\right]-\int_{I} r^{m}\left(\rho^{\gamma}\right)_{r t} u_{t t}+c \int_{I} r^{m}\left(\rho_{t t}^{2}+f_{t}^{2}\right)+c \\
\leq & -\frac{d}{d t} \int_{I}\left[\frac{1}{2} r^{m} \rho_{t} u_{t}^{2}+r^{m}\left(\rho_{t} u u_{r}-\rho_{t} f\right) u_{t}\right]+c\left\|u_{t}\right\|_{L^{\infty}}^{2}\left(\int_{I} r^{m} \rho u_{t}^{2}+\int_{I} r^{m} \rho_{t}^{2}\right) \\
& +c \int_{I}\left(r^{m} u_{r t}^{2}+m r^{m-2} u_{t}^{2}\right)\left[\int_{I} r^{m}\left(\rho_{t t}^{2}+f_{t}^{2}\right)+1\right]-\int_{I} r^{m}\left(\rho^{\gamma}\right)_{r t} u_{t t}+c \int_{I} r^{m}\left(\rho_{t t}^{2}+f_{t}^{2}\right)+c \\
\leq & -\frac{d}{d t} \int_{I}\left[\frac{1}{2} r^{m} \rho_{t} u_{t}^{2}+r^{m}\left(\rho_{t} u u_{r}-\rho_{t} f\right) u_{t}\right]+c \int_{I}\left(r^{m} u_{r t}^{2}+m r^{m-2} u_{t}^{2}\right)\left[\int_{I} r^{m}\left(\rho_{t t}^{2}+f_{t}^{2}\right)+1\right] \\
& -\int_{I} r^{m}\left(\rho^{\gamma}\right)_{r t} u_{t t}+c \int_{I} r^{m}\left(\rho_{t t}^{2}+f_{t}^{2}\right)+c .
\end{aligned}
$$

Integrating the above inequality over $(0, t)$, we have

$$
\begin{aligned}
& \frac{1}{2} \int_{0}^{t} \int_{I} r^{m} \rho u_{t t}^{2}+\frac{1}{2} \int_{I}\left(r^{m} u_{r t}^{2}+m r^{m-2} u_{t}^{2}\right) \\
\leq & -\int_{I}\left[\frac{1}{2} r^{m} \rho_{t} u_{t}^{2}+r^{m}\left(\rho_{t} u u_{r}-\rho_{t} f\right) u_{t}\right]+c \int_{0}^{t} \int_{I}\left(r^{m} u_{r t}^{2}+m r^{m-2} u_{t}^{2}\right)\left[\int_{I} r^{m}\left(\rho_{t t}^{2}+f_{t}^{2}\right)+1\right] \\
& -\int_{0}^{t} \int_{I} r^{m}\left(\rho^{\gamma}\right)_{r t} u_{t t}+c \\
\leq & \int_{I} \frac{1}{2}\left(r^{m} \rho u\right)_{r} u_{t}^{2}+c\left\|u_{t}\right\|_{L^{\infty}}+c \int_{0}^{t} \int_{I}\left(r^{m} u_{r t}^{2}+m r^{m-2} u_{t}^{2}\right) \int_{I} r^{m}\left(\rho_{t t}^{2}+f_{t}^{2}\right) \\
& -\int_{0}^{t} \int_{I} r^{m}\left(\rho^{\gamma}\right)_{r t} u_{t t}+c,
\end{aligned}
$$

where we have used (1.4) 1 , (1.8), (2.3), Lemma 2.1 Cauchy inequality and the following equalities:

$$
\begin{gathered}
\left.\int_{I}\left(r^{m} u_{r t}^{2}+m r^{m-2} u_{t}^{2}\right)\right|_{t=0}=\left.c \int_{\Omega}\left|\nabla \mathbf{u}_{t}\right|^{2}\right|_{t=0}, \\
\left.\mathbf{u}_{t}\right|_{t=0}=\rho_{0}{ }^{-1}\left(L \mathbf{u}_{0}+\rho_{0} \mathbf{f}(0)-\nabla P\left(\rho_{0}\right)-\rho_{0} \mathbf{u}_{0} \cdot \nabla \mathbf{u}_{0}\right) \\
=\mathbf{g}_{1}-\mathbf{u}_{0} \cdot \nabla \mathbf{u}_{0} \in D_{0}^{1} .
\end{gathered}
$$


Using integration by parts, (2.3), (2.4), Hölder inequality, Cauchy inequality and Lemma 2.1, we obtain

$$
\begin{aligned}
& \frac{1}{2} \int_{0}^{t} \int_{I} r^{m} \rho u_{t t}^{2}+\frac{1}{2} \int_{I}\left(r^{m} u_{r t}^{2}+m r^{m-2} u_{t}^{2}\right) \\
\leq & -\int_{I} r^{m} \rho u u_{t} u_{r t}+c\left[\int_{I}\left(r^{m} u_{r t}^{2}+m r^{m-2} u_{t}^{2}\right)\right]^{\frac{1}{2}}+c \int_{0}^{t} \int_{I}\left(r^{m} u_{r t}^{2}+m r^{m-2} u_{t}^{2}\right) \int_{I} r^{m}\left(\rho_{t t}^{2}+f_{t}^{2}\right) \\
& -\int_{0}^{t} \int_{I} r^{m}\left(\rho^{\gamma}\right)_{r t} u_{t t}+c \\
\leq & \frac{1}{4} \int_{I}\left(r^{m} u_{r t}^{2}+m r^{m-2} u_{t}^{2}\right)+c \int_{0}^{t} \int_{I}\left(r^{m} u_{r t}^{2}+m r^{m-2} u_{t}^{2}\right) \int_{I} r^{m}\left(\rho_{t t}^{2}+f_{t}^{2}\right)-\int_{0}^{t} \int_{I} r^{m}\left(\rho^{\gamma}\right)_{r t} u_{t t}+c .
\end{aligned}
$$

Therefore,

$$
\begin{aligned}
& \int_{0}^{t} \int_{I} r^{m} \rho u_{t t}^{2}+\frac{1}{2} \int_{I}\left(r^{m} u_{r t}^{2}+m r^{m-2} u_{t}^{2}\right) \\
\leq & c \int_{0}^{t} \int_{I}\left(r^{m} u_{r t}^{2}+m r^{m-2} u_{t}^{2}\right) \int_{I} r^{m}\left(\rho_{t t}^{2}+f_{t}^{2}\right)-\int_{0}^{t} \int_{I} r^{m}\left(\rho^{\gamma}\right)_{r t} u_{t t}+c .
\end{aligned}
$$

We shall handle the second integral of the right hand side of the above inequality. Using integration by parts twice formally (firstly with respect to $t$ and then with respect to $r$ ), we have

$$
\begin{aligned}
-\int_{0}^{t} \int_{I} r^{m}\left(\rho^{\gamma}\right)_{r t} u_{t t}= & -\int_{I} r^{m}\left(\rho^{\gamma}\right)_{r t} u_{t}+\left.\int_{I} r^{m}\left(\rho^{\gamma}\right)_{r t} u_{t}\right|_{t=0}+\int_{0}^{t} \int_{I} r^{m}\left(\rho^{\gamma}\right)_{r t t} u_{t} \\
\leq & \int_{I} r^{m}\left(\rho^{\gamma}\right)_{t} u_{r t}+m \int_{I} r^{m-1}\left(\rho^{\gamma}\right)_{t} u_{t}-\int_{0}^{t} \int_{I} r^{m}\left(\rho^{\gamma}\right)_{t t} u_{r t} \\
& -m \int_{0}^{t} \int_{I} r^{m-1}\left(\rho^{\gamma}\right)_{t t} u_{t}+c \\
\leq & \frac{1}{4} \int_{I}\left(r^{m} u_{r t}^{2}+m r^{m-2} u_{t}^{2}\right)+c .
\end{aligned}
$$

Rigorously, this process can be done by the usual method (mollification+taking limits). More precisely, we can take place of $\left(\rho^{\gamma}\right)_{t}$ by $J_{\epsilon} * \overline{\left(\rho^{\gamma}\right)_{t}}$, and then take limits $\epsilon \rightarrow 0^{+}$, where $J_{\epsilon}(\cdot)=\frac{1}{\epsilon} J(\dot{\bar{\epsilon}}) ; J(\cdot)$ is the usual mollifier in $\mathbb{R}^{1} ; \overline{\left(\rho^{\gamma}\right)_{t}}$ is an extension of $\left(\rho^{\gamma}\right)_{t}$ w.r.t. $t$, defined as follows

$$
\overline{\left(\rho^{\gamma}\right)_{t}}= \begin{cases}\left(\rho^{\gamma}\right)_{t}(0), & \text { if } t \in[-1,0], \\ \left(\rho^{\gamma}\right)_{t}(t), & \text { if } t \in[0, T], \\ \left(\rho^{\gamma}\right)_{t}(T), & \text { if } t \in[T, T+1] .\end{cases}
$$

Substituting $\left(*_{2}\right)$ into $\left(*_{1}\right)$, and using Gronwall inequality, we get

$$
\int_{Q_{T}} r^{m} \rho u_{t t}^{2}+\int_{I}\left(r^{m} u_{r t}^{2}+r^{m-2} u_{t}^{2}\right) \leq c .
$$

The proof of Lemma 2.2 is complete. 
LEMMA 2.3. For any $0 \leq t \leq T$, it holds

$$
\int_{I} r^{m} u_{r r r}^{2} \leq c
$$

Proof. Differentiating (2.1) with respect to $r$, we get

$u_{r r r}=\rho_{r} u_{t}+\rho u_{r t}+\rho_{r} u u_{r}+\rho u_{r}^{2}+\rho u u_{r r}+\left(\rho^{\gamma}\right)_{r r}-\frac{m u_{r r}}{r}+\frac{2 m\left(r u_{r}-u\right)}{r^{3}}-\rho_{r} f-\rho f_{r}$.

By (2.5), (2.3), (2.4) and Lemma 2.1, we obtain

$$
\begin{aligned}
\int_{I} r^{m} u_{r r r}^{2} & \leq c \int_{I}\left(r^{m} u_{r t}^{2}+m r^{m-2} u_{t}^{2}\right)+c \int_{I} r^{m}\left|\left(\rho^{\gamma}\right)_{r r}\right|^{2}+c \int_{I} r^{m}\left(f^{2}+f_{r}^{2}\right)+c \\
& \leq c .
\end{aligned}
$$

The proof of Lemma 2.3 is complete.

LEMMA 2.4. For any $0 \leq t \leq T$, it holds

$$
\int_{I} r^{m}\left(\rho_{t t}^{2}+\left|\left(\rho^{\gamma}\right)_{t t}\right|^{2}\right)+\int_{Q_{T}} r^{m} u_{r r t}^{2} \leq c .
$$

Proof. By (2.2), (2.3), (2.4), Lemma 2.1 and Lemma 2.2, we get

$$
\begin{aligned}
\int_{Q_{T}} r^{m} u_{r r t}^{2} \leq & c \int_{Q_{T}} r^{m} \rho_{t}^{2} u_{t}^{2}+c \int_{Q_{T}} r^{m} \rho^{2} u_{t t}^{2}+c \int_{Q_{T}} r^{m} \rho_{t}^{2} u^{2} u_{r}^{2}+c \int_{Q_{T}} r^{m} \rho^{2} u_{t}^{2} u_{r}^{2} \\
& +c \int_{Q_{T}} r^{m} \rho^{2} u^{2} u_{r t}^{2}+c \int_{Q_{T}} r^{m}\left|\left(\rho^{\gamma}\right)_{r t}\right|^{2}+c \int_{Q_{T}}\left(r^{m} u_{r t}^{2}+m r^{m-2} u_{t}^{2}\right) \\
& +c \int_{Q_{T}} r^{m}\left(\rho_{t}^{2} f^{2}+\rho^{2} f_{t}^{2}\right) \\
\leq & c .
\end{aligned}
$$

Multiplying (1.4) 1 by $\gamma \rho^{\gamma-1}$, we get

$$
\left(\rho^{\gamma}\right)_{t}+\gamma \rho^{\gamma} u_{r}+\left(\rho^{\gamma}\right)_{r} u+m \gamma r^{-1} \rho^{\gamma} u=0
$$

(1.4) 1 , (2.3), (2.4), (2.6), Lemma 2.1 and Lemma 2.2 imply

$$
\int_{I} r^{m}\left(\rho_{t t}^{2}+\left|\left(\rho^{\gamma}\right)_{t t}\right|^{2}\right) \leq c
$$

This proves Lemma 2.4

To sum up, we get

$$
\left\|\left(\rho, \rho^{\gamma}\right)\right\|_{H_{r}^{2}}+\left\|\left(\rho_{t},\left(\rho^{\gamma}\right)_{t}\right)\right\|_{H_{r}^{1}}+\left\|\left(\rho_{t t},\left(\rho^{\gamma}\right)_{t t}\right)\right\|_{L_{r}^{2}} \leq c, \rho \geq \frac{\delta}{c}
$$

and

$$
\int_{I} r^{m}\left(u_{r t}^{2}+r^{-2} u_{t}^{2}+u_{r r r}^{2}+u_{r r}^{2}+u_{r}^{2}+r^{-2} u^{2}\right)+\int_{Q_{T}} r^{m}\left(\rho u_{t t}^{2}+u_{r r t}^{2}\right) \leq c
$$


By (2.7) and (2.8), we complete the proof of Theorem 2.1.

Proof of Theorem 1.1

Let $b<\infty$, and denote $\rho_{0}^{\delta}=\rho_{0}+\delta$, for $\delta \in(0,1)$, we have

$$
\begin{gathered}
\rho_{0}^{\delta} \rightarrow \rho_{0}, \quad \text { in } H^{2}(I), \\
\left(\rho_{0}^{\delta}\right)^{\gamma} \rightarrow \rho_{0}^{\gamma}, \text { in } H^{2}(I) .
\end{gathered}
$$

Let $u_{0}^{\delta}$ be the unique solution to the equation:

$$
\left(u_{0 r}^{\delta}+\frac{m u_{0}^{\delta}}{r}\right)_{r}-\left[\left(\rho_{0}^{\delta}\right)^{\gamma}\right]_{r}=\rho_{0}^{\delta}\left[g_{1}-f(0)\right], \text { in } I,
$$

for $\left.u_{0}^{\delta}\right|_{\partial I}=0$. (1.8) implies

$$
\left(u_{0 r}+\frac{m u_{0}}{r}\right)_{r}-\left[\left(\rho_{0}\right)^{\gamma}\right]_{r}=\rho_{0}\left[g_{1}-f(0)\right], \text { in } I,
$$

for $\left.u_{0}\right|_{\partial I}=0$.

From (2.9)-(2.12) and the standard elliptic estimates, we obtain

$$
u_{0}^{\delta} \rightarrow u_{0}, \quad \text { in } H^{3}(I), \text { as } \delta \rightarrow 0 .
$$

Consider (1.4)-(1.6) with initial-boundary data replaced by

$$
\left.\left(\rho^{\delta}, u^{\delta}\right)\right|_{t=0}=\left(\rho_{0}^{\delta}, u_{0}^{\delta}\right), \text { in } I,
$$

and

$$
\left.u^{\delta}\right|_{\partial I}=0, \text { for } t \geq 0 .
$$

Then we get a unique solution $\left(\rho^{\delta}, u^{\delta}\right)$ for each $\delta>0$ by Theorem 2.1 .

Following the estimates in the proofs of Theorem 2.1. we can also get (2.7) and (2.8) with $(\rho, u)$ replaced by $\left(\rho^{\delta}, u^{\delta}\right)$. Since $b<\infty$ and $a>0$, it follows from (2.7) and (2.8) that

$$
\left\|\left(\rho^{\delta},\left(\rho^{\delta}\right)^{\gamma}\right)\right\|_{H^{2}(I)}+\left\|\left(\rho_{t}^{\delta},\left(\left(\rho^{\delta}\right)^{\gamma}\right)_{t}\right)\right\|_{H^{1}(I)}+\left\|\left(\rho_{t t}^{\delta},\left(\left(\rho^{\delta}\right)^{\gamma}\right)_{t t}\right)\right\|_{L^{2}(I)} \leq c,
$$

and

$$
\rho^{\delta} \geq \frac{\delta}{c},\left\|u_{t}^{\delta}\right\|_{H^{1}(I)}+\left\|u^{\delta}\right\|_{H^{3}(I)}+\int_{Q_{T}}\left(\rho^{\delta}\left|u_{t t}^{\delta}\right|^{2}+\left|u_{r r t}^{\delta}\right|^{2}\right) \leq c(b) .
$$

Based on the estimates in (2.14) and (2.15), we get a solution $(\rho, u)$ to (1.4)-(1.6) after taking the limit $\delta \rightarrow 0$ (take the subsequence if necessary), satisfying

$$
\left\{\begin{array}{l}
\left(\rho, \rho^{\gamma}\right) \in L^{\infty}\left([0, T] ; H^{2}(I)\right), \quad\left(\rho_{t},\left(\rho^{\gamma}\right)_{t}\right) \in L^{\infty}\left([0, T] ; H^{1}(I)\right), \\
\left(\rho_{t t},\left(\rho^{\gamma}\right)_{t t}\right) \in L^{\infty}\left([0, T] ; L^{2}(I)\right), \quad \rho \geq 0, \quad\left(\rho u_{t}\right)_{t} \in L^{2}\left([0, T] ; L^{2}(I)\right), \\
u \in L^{\infty}\left([0, T] ; H^{3}(I) \cap H_{0}^{1}(I)\right), \quad u_{t} \in L^{\infty}\left([0, T] ; H_{0}^{1}(I)\right) \cap L^{2}\left([0, T] ; H^{2}(I)\right) .
\end{array}\right.
$$


Since $u \in L^{\infty}\left([0, T] ; H^{3}(I)\right)$ and $u_{t} \in L^{\infty}\left([0, T] ; H_{0}^{1}(I)\right)$, then we get $u \in C\left([0, T] ; H^{2}(I)\right)$ (refer to [7). By (1.4) $1,(2.6)$ and similar arguments as in [4, 5], we get

$$
\rho \in C\left([0, T] ; H^{2}(I)\right), \rho_{t} \in C\left([0, T] ; H^{1}(I)\right),
$$

and

$$
\rho^{\gamma} \in C\left([0, T] ; H^{2}(I)\right),\left(\rho^{\gamma}\right)_{t} \in C\left([0, T] ; H^{1}(I)\right) .
$$

Denote $G=\left(u_{r}+\frac{m u}{r}-\rho^{\gamma}\right)_{r}+\rho f$. By (2.1) and (2.16), we have

$$
\begin{gathered}
G=\rho u_{t}+\rho u u_{r} \in L^{2}\left([0, T] ; H^{2}(I)\right), \\
G_{t}=\left(\rho u_{t}+\rho u u_{r}\right)_{t} \in L^{2}\left([0, T] ; L^{2}(I)\right) .
\end{gathered}
$$

By the embedding theorem ([7]), we have $G \in C\left([0, T] ; H^{1}(I)\right)$. Since $\rho f \in C\left([0, T] ; H^{1}(I)\right)$, we get

$$
\left(u_{r}+\frac{m u}{r}-\rho^{\gamma}\right)_{r} \in C\left([0, T] ; H^{1}(I)\right) .
$$

This means

$$
u_{r}+\frac{m u}{r}-\rho^{\gamma} \in C\left([0, T] ; H^{2}(I)\right) .
$$

By (2.18) and (2.19), we get

$$
u_{r}+\frac{m u}{r} \in C\left([0, T] ; H^{2}\right) .
$$

This together with $u \in C\left([0, T] ; H^{2}(I)\right)$ implies

$$
u \in C\left([0, T] ; H^{3}(I)\right) .
$$

(1.4) $2,(2.17),(2.18)$ and (2.20) give

$$
(\rho u)_{t} \in C\left([0, T] ; H^{1}(I)\right) .
$$

Denote

$$
\rho(\mathbf{x}, t)=\rho(r, t), \mathbf{u}(\mathbf{x}, t)=u(r, t) \frac{\mathbf{x}}{r} .
$$

It follows from (2.16)-(2.18) and (2.20)-(2.22), we complete the proof of Theorem 1.1 for $b<\infty$. For $b=\infty$, we can use the similar methods as in 3 together with the estimates (2.7) and (2.8) uniform for $b$ to get it. We omit details here for simplicity.

3. Proof of Theorem 1.2. Similar to the proof of Theorem 1.1 we need the following auxiliary theorem.

TheOrem 3.1. Consider the same assumptions as in Theorem 1.2, and in addition assume that $\rho_{0} \geq \delta>0$ and $b<\infty$. Then for any $T>0$, there exists a unique global classical solution $(\rho, u)$ to $(1.4)$-(1.6) satisfying

$$
\rho \in C\left([0, T] ; H^{5}(I)\right), \rho \geq \frac{\delta}{c}, u \in C\left([0, T] ; H^{5}(I) \cap H_{0}^{1}(I)\right) \cap L^{2}\left([0, T] ; H^{6}(I)\right),
$$




$$
\begin{gathered}
u_{t} \in C\left([0, T] ; H^{3}(I) \cap H_{0}^{1}(I)\right) \cap L^{2}\left([0, T] ; H^{4}(I)\right), \\
u_{t t} \in C\left([0, T] ; H_{0}^{1}(I)\right) \cap L^{2}\left([0, T] ; H^{2}(I)\right), u_{t t t} \in L^{2}\left([0, T] ; L^{2}(I)\right) .
\end{gathered}
$$

The proof of local existence of the solutions as in Theorem 3.1 can be done by the successive approximations as in [5] and references therein, together with the estimates in Section 3 for higher order derivatives of the solutions. We omit it here for brevity. Therefore, Theorem 3.1 can be proved by some a priori estimates globally in time. Since (2.7) and (2.8) are also valid here, we need some other a priori estimates about higher order derivatives of $(\rho, u)$. The generic positive constant $c$ may depend on the initial data presented in Theorem 1.2 and other known constants but independent of $\delta$ and $b$.

LEMma 3.1. For any $0 \leq t \leq T$, it holds

$$
\int_{I} r^{m}\left[\rho_{r r r}^{2}+\rho_{r r t}^{2}+\left|\left(\rho^{\gamma}\right)_{r r r}\right|^{2}+\left|\left(\rho^{\gamma}\right)_{r r t}\right|^{2}\right]+\int_{Q_{T}} r^{m}\left[\rho_{r t t}^{2}+\left|\left(\rho^{\gamma}\right)_{r t t}\right|^{2}+u_{r r r r}^{2}\right] \leq c .
$$

Proof. Taking derivative of order three on both sides of $(1.4)_{1}$ with respect to $r$, multiplying it by $r^{m} \rho_{r r r}$, and integrating by parts over $I$, we have

$$
\begin{gathered}
\frac{1}{2} \frac{d}{d t} \int_{I} r^{m} \rho_{r r r}^{2}=\int_{I} r^{m} \rho_{r r r}\left[-m r^{-1} \rho u_{r r r}-3 m r^{-1} \rho_{r} u_{r r}-3 m r^{-1} \rho_{r r} u_{r}+\frac{3 m \rho u_{r r}}{r^{2}}\right. \\
-\frac{6 m \rho u_{r}}{r^{3}}-m r^{-1} \rho_{r r r} u+\frac{3 m \rho_{r r} u}{r^{2}}+\frac{6 m \rho_{r} u_{r}}{r^{2}}-\frac{6 m \rho_{r} u}{r^{3}}+\frac{6 m \rho u}{r^{4}}-4 \rho_{r r r} u_{r}-6 \rho_{r r} u_{r r} \\
\left.-4 \rho_{r} u_{r r r}\right]+\frac{1}{2} \int_{I} r^{m} \rho_{r r r}^{2} u_{r}+\frac{1}{2} \int_{I} m r^{m-1} \rho_{r r r}^{2} u-\int_{I} r^{m} \rho \rho_{r r r} u_{r r r r} .
\end{gathered}
$$

By Sobolev inequality, (2.3), (2.4) (2.7), (2.8) and Cauchy inequality, we get

$$
\frac{d}{d t} \int_{I} r^{m} \rho_{r r r}^{2} \leq c \int_{I} r^{m} \rho_{r r r}^{2}+c \int_{I} r^{m} u_{r r r r}^{2}+c .
$$

Similarly to (3.1), we get from (2.6)

$$
\frac{d}{d t} \int_{I} r^{m}\left|\left(\rho^{\gamma}\right)_{r r r}\right|^{2} \leq c \int_{I} r^{m}\left|\left(\rho^{\gamma}\right)_{r r r}\right|^{2}+c \int_{I} r^{m} u_{r r r r}^{2}+c .
$$

By (3.1) and (3.2), we have

$$
\frac{d}{d t} \int_{I} r^{m}\left(\rho_{r r r}^{2}+\left|\left(\rho^{\gamma}\right)_{r r r}\right|^{2}\right) \leq c \int_{I} r^{m}\left(\rho_{r r r}^{2}+\left|\left(\rho^{\gamma}\right)_{r r r}\right|^{2}\right)+c \int_{I} r^{m} u_{r r r r}^{2}+c .
$$

Differentiating (2.5) with respect to $r$, we have

$$
\begin{aligned}
u_{r r r r}= & \rho_{r r} u_{t}+2 \rho_{r} u_{r t}+\rho u_{r r t}+\left(\rho_{r} u u_{r}+\rho u_{r}^{2}+\rho u u_{r r}\right)_{r}+\left(\rho^{\gamma}\right)_{r r r}-m r^{-1} u_{r r r} \\
& +3 m r^{-2} u_{r r}-6 m r^{-3} u_{r}+\frac{6 m u}{r^{4}}-\rho_{r r} f-2 \rho_{r} f_{r}-\rho f_{r r} .
\end{aligned}
$$

From (3.4), (2.3), (2.4), (2.7) and (2.8), we obtain

$$
\int_{I} r^{m} u_{r r r r}^{2} \leq c \int_{I} r^{m}\left|\left(\rho^{\gamma}\right)_{r r r}\right|^{2}+c \int_{I} r^{m} \rho u_{r r t}^{2}+c \int_{I} r^{m} f_{r r}^{2}+c .
$$

By (3.3), (3.5), (2.4) and (2.7), we get

$$
\frac{d}{d t} \int_{I} r^{m}\left(\rho_{r r r}^{2}+\left|\left(\rho^{\gamma}\right)_{r r r}\right|^{2}\right) \leq c \int_{I} r^{m}\left(\rho_{r r r}^{2}+\left|\left(\rho^{\gamma}\right)_{r r r}\right|^{2}\right)+c \int_{I} r^{m} u_{r r t}^{2}+c \int_{I} r^{m} f_{r r}^{2}+c .
$$


By Gronwall inequality and (2.8), we obtain

$$
\int_{I} r^{m}\left(\rho_{r r r}^{2}+\left|\left(\rho^{\gamma}\right)_{r r r}\right|^{2}\right) \leq c .
$$

It follows from (1.4) $1,(2.3),(2.4),(2.6),(2.7),(2.8),(3.5)$ and (3.6) that

$$
\int_{I} r^{m}\left[\rho_{r r t}^{2}+\left|\left(\rho^{\gamma}\right)_{r r t}\right|^{2}\right]+\int_{Q_{T}} r^{m}\left[\rho_{r t t}^{2}+\left|\left(\rho^{\gamma}\right)_{r t t}\right|^{2}+u_{r r r r}^{2}\right] \leq c .
$$

The proof of Lemma 3.1 is complete.

Lemma 3.2. For any $T>0$, we have

$$
\left\|(\sqrt{\rho})_{r}\right\|_{L^{\infty}\left(Q_{T}\right)}+\left\|(\sqrt{\rho})_{t}\right\|_{L^{\infty}\left(Q_{T}\right)} \leq c .
$$

Proof. Multiplying (1.4) 1 by $\frac{1}{2 \sqrt{\rho}}$, we have

$$
(\sqrt{\rho})_{t}+\frac{m r^{-1}}{2} \sqrt{\rho} u+\frac{1}{2} \sqrt{\rho} u_{r}+(\sqrt{\rho})_{r} u=0 .
$$

Differentiating (3.7) with respect to $r$, we get

$(\sqrt{\rho})_{r t}+\frac{m r^{-1}}{2}(\sqrt{\rho})_{r} u+\frac{m r^{-1}}{2} \sqrt{\rho} u_{r}-\frac{m \sqrt{\rho} u}{2 r^{2}}+\frac{3}{2}(\sqrt{\rho})_{r} u_{r}+\frac{1}{2} \sqrt{\rho} u_{r r}+(\sqrt{\rho})_{r r} u=0$.

Denote $h=(\sqrt{\rho})_{r}$, we have

$$
h_{t}+h_{r} u+h\left(\frac{m r^{-1}}{2} u+\frac{3}{2} u_{r}\right)+\frac{m r^{-1}}{2} \sqrt{\rho} u_{r}-\frac{m \sqrt{\rho} u}{2 r^{2}}+\frac{1}{2} \sqrt{\rho} u_{r r}=0,
$$

which implies

$$
\begin{aligned}
& \frac{d}{d t}\left\{h \exp \left[\int_{0}^{t}\left(\frac{m r^{-1}}{2} u+\frac{3}{2} u_{r}\right)(r(\tau, y), \tau) d \tau\right]\right\} \\
&=-\left(\frac{m r^{-1} \sqrt{\rho} u_{r}}{2}-\frac{m \sqrt{\rho} u}{2 r^{2}}+\frac{\sqrt{\rho} u_{r r}}{2}\right) \\
& \quad \times \exp \left[\int_{0}^{t}\left(\frac{m r^{-1} u}{2}+\frac{3 u_{r}}{2}\right)(r(\tau, y), \tau) d \tau\right],
\end{aligned}
$$

where $r(t, y)$ satisfies

$$
\left\{\begin{array}{l}
\frac{d r(t, y)}{d t}=u(r(t, y), t), 0 \leq t<s \\
r(s, y)=y
\end{array}\right.
$$

Integrating (3.8) over $(0, s)$, we get

$$
\begin{aligned}
h(y, s)=\exp (- & \left.\int_{0}^{s}\left(\frac{m r^{-1} u}{2}+\frac{3 u_{r}}{2}\right)(r(\tau, y), \tau) d \tau\right) h(r(0, y), 0) \\
-\int_{0}^{s}[ & \left(\frac{m r^{-1} \sqrt{\rho} u_{r}}{2}-\frac{m \sqrt{\rho} u}{2 r^{2}}+\frac{\sqrt{\rho} u_{r r}}{2}\right) \\
& \left.\times \exp \left(\int_{s}^{t}\left(\frac{m r^{-1} u}{2}+\frac{3 u_{r}}{2}\right)(r(\tau, y), \tau) d \tau\right)\right] d t .
\end{aligned}
$$


This together with (2.3), (2.4), (2.7) and (2.8) implies

$$
\left\|(\sqrt{\rho})_{r}\right\|_{L^{\infty}\left(Q_{T}\right)} \leq c .
$$

From (3.7), (3.9), (2.3), (2.4), (2.7) and (2.8), we get

$$
\left\|(\sqrt{\rho})_{t}\right\|_{L^{\infty}\left(Q_{T}\right)} \leq c .
$$

The proof of Lemma 3.2 is complete.

Lemma 3.3. For any $0 \leq t \leq T$, it holds

$$
\int_{I} r^{m}\left(\rho^{3} u_{t t}^{2}+\rho u_{r r t}^{2}+u_{r r r r}^{2}\right)+\int_{Q_{T}} r^{m}\left(\rho^{2} u_{r t t}^{2}+u_{r r r t}^{2}\right) \leq c .
$$

Proof. Differentiating (2.2) with respect to $t$, multiplying it by $r^{m} \rho^{2} u_{t t}$, and integrating by parts over $I$, we have

$$
\begin{aligned}
& \frac{1}{2} \frac{d}{d t} \int_{I} r^{m} \rho^{3} u_{t t}^{2}+\int_{I}\left(r^{m} \rho^{2} u_{r t t}^{2}+m r^{m-2} \rho^{2} u_{t t}^{2}\right) \\
= & -\frac{1}{2} \int_{I} r^{m} \rho^{2} \rho_{t} u_{t t}^{2}-\int_{I} r^{m} \rho^{2} u_{t t}\left[\rho_{t t} u_{t}+\rho_{t t} u u_{r}+2 \rho_{t} u_{t} u_{r}+2 \rho_{t} u u_{r t}+\rho u_{t t} u_{r}\right. \\
& \left.+2 \rho u_{t} u_{r t}+\rho u u_{r t t}+\left(\rho^{\gamma}\right)_{r t t}\right]-2 \int_{I} r^{m} \rho \rho_{r} u_{t t} u_{r t t}+\int_{I} r^{m} \rho^{2} u_{t t}\left(\rho_{t t} f+2 \rho_{t} f_{t}+\rho f_{t t}\right) \\
\leq & c \int_{I} r^{m} \rho u_{t t}^{2}+\frac{1}{4} \int_{I} r^{m} \rho^{2} u_{r t t}^{2}+c \int_{I} r^{m}\left|\left(\rho^{\gamma}\right)_{r t t}\right|^{2}-4 \int_{I} r^{m} \rho u_{r t t} \sqrt{\rho} u_{t t}(\sqrt{\rho})_{r} \\
& +c \int_{I} r^{m}\left(\rho_{t t}^{2} f^{2}+\rho_{t}^{2} f_{t}^{2}+\rho^{2} f_{t t}^{2}\right)+c \\
\leq & c \int_{I} r^{m} \rho u_{t t}^{2}+\frac{1}{4} \int_{I} r^{m} \rho^{2} u_{r t t}^{2}+c \int_{I} r^{m}\left|\left(\rho^{\gamma}\right)_{r t t}\right|^{2}+\frac{1}{4} \int_{I} r^{m} \rho^{2} u_{r t t}^{2}+\int_{I} r^{m} f_{t t}^{2}+c,
\end{aligned}
$$

where we have used (2.3), (2.4), (2.7), (2.8), Lemma 3.2 and Cauchy inequality.

Thus,

$$
\begin{aligned}
& \frac{d}{d t} \int_{I} r^{m} \rho^{3} u_{t t}^{2}+\int_{I}\left(r^{m} \rho^{2} u_{r t t}^{2}+m r^{m-2} \rho^{2} u_{t t}^{2}\right) \\
\leq & c \int_{I} r^{m} \rho u_{t t}^{2}+c \int_{I} r^{m}\left|\left(\rho^{\gamma}\right)_{r t t}\right|^{2}+\int_{I} r^{m} f_{t t}^{2}+c .
\end{aligned}
$$

Integrating (3.10) over $(0, t)$, and using (2.8) and Lemma 3.1, we get

$$
\int_{I} r^{m} \rho^{3} u_{t t}^{2}(t)+\int_{0}^{t} \int_{I}\left(r^{m} \rho^{2} u_{r t t}^{2}+m r^{m-2} \rho^{2} u_{t t}^{2}\right) \leq \int_{I} r^{m} \rho^{3} u_{t t}^{2}(0)+c .
$$

By (2.1), (2.2), (1.8) and $\sqrt{\rho_{0}} \nabla^{2} \mathbf{g}_{1} \in L^{2}$, we have

$$
\int_{I} r^{m} \rho^{3} u_{t t}^{2}(0) \leq c
$$

(3.11) and (3.12) give

$$
\int_{I} r^{m} \rho^{3} u_{t t}^{2}+\int_{Q_{T}}\left(r^{m} \rho^{2} u_{r t t}^{2}+r^{m-2} \rho^{2} u_{t t}^{2}\right) \leq c .
$$


By (2.2), Cauchy inequality, (2.3), (2.4), (2.7), (2.8) and (3.13), we have

$$
\begin{aligned}
\int_{I} r^{m} \rho u_{r r t}^{2} \leq & c \int_{I} r^{m} \rho \rho_{t}^{2} u_{t}^{2}+c \int_{I} r^{m} \rho^{3} u_{t t}^{2}+c \int_{I} r^{m} \rho \rho_{t}^{2} u^{2} u_{r}^{2}+c \int_{I} r^{m} \rho^{3} u_{t}^{2} u_{r}^{2} \\
& +c \int_{I} r^{m} \rho^{3} u^{2} u_{r t}^{2}+c \int_{I} r^{m} \rho\left|\left(\rho^{\gamma}\right)_{r t}\right|^{2}+c \int_{I} r^{m-2} \rho u_{r t}^{2}+c \int_{I} r^{m-4} \rho u_{t}^{2} \\
& +c \int_{I} r^{m} \rho \rho_{t}^{2} f^{2}+c \int_{I} r^{m} \rho^{3} f_{t}^{2} \\
\leq & c .
\end{aligned}
$$

Differentiating (2.2) with respect to $r$, we get

$$
\begin{aligned}
u_{r r r t}= & \rho_{r t} u_{t}+2(\sqrt{\rho})_{r} \sqrt{\rho} u_{t t}+\rho_{t} u_{r t}+\rho u_{r t t}+\rho_{r t} u u_{r}+\rho_{r} u_{t} u_{r}+\rho_{r} u u_{r t}+\rho_{t} u_{r}^{2} \\
& +2 \rho u_{r} u_{r t}+\rho_{t} u u_{r r}+\rho u_{t} u_{r r}+\rho u u_{r r t}+\left(\rho^{\gamma}\right)_{r r t}-m r^{-1} u_{r r t}+2 m r^{-2} u_{r t} \\
& -\frac{2 m u_{t}}{r^{3}}-\rho_{r t} f-\rho_{t} f_{r}-\rho_{r} f_{t}-\rho f_{r t} .
\end{aligned}
$$

By (3.13), (3.15), (2.3), (2.4), (2.7), (2.8), Lemma 3.1 and Lemma 3.2, we have

$$
\int_{Q_{T}} r^{m} u_{r r r t}^{2} \leq c .
$$

(3.5), (3.14) and Lemma 3.1 immediately give

$$
\int_{I} r^{m} u_{r r r r}^{2} \leq c .
$$

The proof of Lemma 3.3 is complete.

LEMMA 3.4. For any $0 \leq t \leq T$, it holds

$$
\int_{I} r^{m}\left[\rho_{r r r r}^{2}+\left|\left(\rho^{\gamma}\right)_{r r r r}\right|^{2}\right]+\int_{Q_{T}} r^{m} u_{r r r r r}^{2} \leq c .
$$

Proof. Differentiating (1.4) 1 four times with respect to $r$, we get

$$
\begin{aligned}
& \rho_{r r r r t}+m r^{-1} \rho u_{r r r r}+4 m r^{-1} \rho_{r} u_{r r r}+6 m r^{-1} \rho_{r r} u_{r r}-\frac{4 m \rho u_{r r r}}{r^{2}}+\frac{12 m \rho u_{r r}}{r^{3}}-\frac{18 m \rho u_{r}}{r^{4}} \\
& +4 m r^{-1} \rho_{r r r} u_{r}+m r^{-1} \rho_{r r r r} u-\frac{4 m \rho_{r r r} u}{r^{2}}-\frac{12 m \rho_{r} u_{r r}}{r^{2}}-\frac{12 m \rho_{r r} u_{r}}{r^{2}}+\frac{24 m \rho_{r} u_{r}}{r^{3}} \\
& +\frac{12 m \rho_{r r} u}{r^{3}}-\frac{6 m \rho u_{r}}{r^{4}}-\frac{24 m \rho_{r} u}{r^{4}}+\frac{24 m \rho u}{r^{5}}+\rho u_{r r r r r}+5 \rho_{r} u_{r r r r} \\
& +10 \rho_{r r} u_{r r r}+10 \rho_{r r r} u_{r r}+5 \rho_{r r r} u_{r}+\rho_{r r r r r} u=0
\end{aligned}
$$

Multiplying (3.16) by $r^{m} \rho_{r r r r}$, integrating over $I$, and using integration by parts, (2.3), (2.4), (2.7), (2.8), Lemma 3.1, Lemma 3.3, we get

$$
\frac{d}{d t} \int_{I} r^{m} \rho_{r r r r}^{2} \leq c \int_{I} r^{m} \rho_{r r r r}^{2}+c \int_{I} r^{m} u_{r r r r r}^{2}+c .
$$

Similarly, we have

$$
\frac{d}{d t} \int_{I} r^{m}\left|\left(\rho^{\gamma}\right)_{r r r r}\right|^{2} \leq c \int_{I} r^{m}\left|\left(\rho^{\gamma}\right)_{r r r r}\right|^{2}+c \int_{I} r^{m} u_{r r r r r}^{2}+c .
$$


By (3.17) and (3.18), we obtain

$$
\frac{d}{d t} \int_{I} r^{m}\left(\rho_{r r r r}^{2}+\left|\left(\rho^{\gamma}\right)_{r r r r}\right|^{2}\right) \leq c \int_{I} r^{m}\left(\rho_{r r r r}^{2}+\left|\left(\rho^{\gamma}\right)_{r r r r}\right|^{2}\right)+c \int_{I} r^{m} u_{r r r r}^{2}+c .
$$

Now we estimate $\int_{I} r^{m} u_{r r r r}^{2}$. Differentiating (3.4) with respect to $r$, we have

$$
\begin{aligned}
u_{r r r r r}= & \rho_{r r r} u_{t}+3 \rho_{r r} u_{r t}+3 \rho_{r} u_{r r t}+\rho u_{r r r t}+\left(\rho_{r} u u_{r}+\rho u_{r}^{2}+\rho u u_{r r}\right)_{r r}+\left(\rho^{\gamma}\right)_{r r r r} \\
& -m r^{-1} u_{r r r r}+4 m r^{-2} u_{r r r}-12 m r^{-3} u_{r r}+24 m r^{-4} u_{r}-\frac{24 m u}{r^{5}} \\
& -\rho_{r r r} f-3 \rho_{r r} f_{r}-3 \rho_{r} f_{r r}-\rho f_{r r r} .
\end{aligned}
$$

It follows from (3.20), (2.3), (2.4), (2.7), (2.8), Lemma 3.1 and Lemma 3.3 that

$$
\begin{aligned}
\int_{I} r^{m} u_{r r r r r}^{2} \leq & c \int_{I} r^{m} \rho_{r}^{2} u_{r r t}^{2}+c \int_{I} r^{m} \rho^{2} u_{r r r t}^{2}+c \int_{I} r^{m}\left|\left(\rho^{\gamma}\right)_{r r r r}\right|^{2} \\
& +c \int_{I} r^{m}\left(f_{r}^{2}+f_{r r}^{2}+f_{r r r}^{2}\right)+c .
\end{aligned}
$$

Since

$$
\int_{I} r^{m} \rho_{r}^{2} u_{r r t}^{2}=4 \int_{I} r^{m} \rho\left|(\sqrt{\rho})_{r}\right|^{2} u_{r r t}^{2}
$$

This together with (2.7), (2.8), Lemma 3.2 and Lemma 3.3 gives

$$
\int_{I} r^{m} u_{r r r r r}^{2} \leq c \int_{I} r^{m} \rho^{2} u_{r r r t}^{2}+c \int_{I} r^{m}\left|\left(\rho^{\gamma}\right)_{r r r r}\right|^{2}+c \int_{I} r^{m}\left(f_{r}^{2}+f_{r r}^{2}+f_{r r r}^{2}\right)+c
$$

Substituting (3.22) into (3.19), we obtain

$$
\begin{aligned}
& \frac{d}{d t} \int_{I} r^{m}\left(\rho_{r r r r}^{2}+\left|\left(\rho^{\gamma}\right)_{r r r r}\right|^{2}\right) \\
\leq & c \int_{I} r^{m}\left(\rho_{r r r r}^{2}+\left|\left(\rho^{\gamma}\right)_{r r r r}\right|^{2}\right)+c \int_{I} r^{m} u_{r r r t}^{2}+c \int_{I} r^{m}\left(f_{r}^{2}+f_{r r}^{2}+f_{r r r}^{2}\right)+c .
\end{aligned}
$$

Using Gronwall inequality and Lemma 3.3. we get

$$
\int_{I} r^{m}\left(\rho_{r r r r}^{2}+\left|\left(\rho^{\gamma}\right)_{r r r r}\right|^{2}\right) \leq c
$$

It follows from (3.22), (3.23) and Lemma 3.3 that

$$
\int_{Q_{T}} r^{m} u_{r r r r r}^{2} \leq c
$$

This proves Lemma 3.4 .

From (1.4) 1 , (2.3), (2.4), (2.6), (2.7), (2.8) and Lemmas 3.1 3.4, we immediately get the following estimate.

LEMMA 3.5. For any $0 \leq t \leq T$, it holds

$$
\int_{I} r^{m}\left[\rho_{r t t}^{2}+\left|\left(\rho^{\gamma}\right)_{r t t}\right|^{2}+\rho_{r r r t}^{2}+\left|\left(\rho^{\gamma}\right)_{r r r t}\right|^{2}\right]
$$




$$
+\int_{Q_{T}} r^{m}\left[\rho_{t t t}^{2}+\left|\left(\rho^{\gamma}\right)_{t t t}\right|^{2}+\rho_{r r t t}^{2}+\left|\left(\rho^{\gamma}\right)_{r r t t}\right|^{2}\right] \leq c .
$$

Lemma 3.6. For any $0 \leq t \leq T$, it holds

$$
\int_{I}\left(r^{m} \rho^{4} u_{r t t}^{2}+r^{m-2} \rho^{4} u_{t t}^{2}\right)+\int_{Q_{T}} r^{m} \rho^{5} u_{t t t}^{2} \leq c .
$$

Proof. Differentiating (2.2) with respect to $t$, multiplying it by $r^{m} \rho^{4} u_{t t t}$, and integrating by parts over $I$, we have

$$
\begin{aligned}
& \int_{I} r^{m} \rho^{5} u_{t t t}^{2}+\frac{1}{2} \frac{d}{d t} \int_{I}\left(r^{m} \rho^{4} u_{r t t}^{2}+m r^{m-2} \rho^{4} u_{t t}^{2}\right) \\
= & 2 \int_{I} r^{m} \rho^{3} \rho_{t} u_{r t t}^{2}-4 \int_{I} r^{m} \rho^{3} \rho_{r} u_{r t t} u_{t t t}-\int_{I} r^{m} \rho^{4} u_{t t t}\left[\rho_{t t} u_{t}+2 \rho_{t} u_{t t}+\rho_{t t} u u_{r}+2 \rho_{t} u_{t} u_{r}\right. \\
& \left.+2 \rho_{t} u u_{r t}+\rho u_{t t} u_{r}+2 \rho u_{t} u_{r t}+\rho u u_{r t t}+\left(\rho^{\gamma}\right)_{r t t}\right]+2 \int_{I} m r^{m-2} \rho^{3} \rho_{t} u_{t t}^{2} \\
& +\int_{I} r^{m} \rho^{4} u_{t t t}\left(\rho_{t t} f+2 \rho_{t} f_{t}+\rho f_{t t}\right) \\
\leq & c \int_{I} r^{m} \rho^{2} u_{r t t}^{2}-8 \int_{I} r^{m} \rho^{\frac{5}{2}} u_{t t t} \rho(\sqrt{\rho})_{r} u_{r t t}+\frac{1}{4} \int_{I} r^{m} \rho^{5} u_{t t t}^{2}+c+c \int_{I} r^{m}\left(f^{2}+f_{t}^{2}+f_{t t}^{2}\right) \\
\leq & c \int_{I} r^{m} \rho^{2} u_{r t t}^{2}+\frac{1}{2} \int_{I} r^{m} \rho^{5} u_{t t t}^{2}+c \int_{I} r^{m}\left(f^{2}+f_{t}^{2}+f_{t t}^{2}\right)+c,
\end{aligned}
$$

where we have used (2.3), (2.4), (2.7), (2.8), Lemma 3.2. Lemma 3.3. Lemma 3.5 and Cauchy inequality.

Thus,

$$
\int_{I} r^{m} \rho^{5} u_{t t t}^{2}+\frac{d}{d t} \int_{I}\left(r^{m} \rho^{4} u_{r t t}^{2}+m r^{m-2} \rho^{4} u_{t t}^{2}\right) \leq c \int_{I} r^{m} \rho^{2} u_{r t t}^{2}+c \int_{I} r^{m}\left(f^{2}+f_{t}^{2}+f_{t t}^{2}\right)+c .
$$

By (1.8), (2.2) and $\rho_{0} \nabla^{3} \mathbf{g}_{1} \in L^{2}$, we have

$$
\int_{Q_{T}} r^{m} \rho^{5} u_{t t t}^{2}+\int_{I}\left(r^{m} \rho^{4} u_{r t t}^{2}+r^{m-2} \rho^{4} u_{t t}^{2}\right) \leq c .
$$

The proof of Lemma 3.6 is complete.

Lemma 3.7. For any $0 \leq t \leq T$, it holds

$$
\int_{I} r^{m}\left(\rho^{2} u_{r r r t}^{2}+\left|\partial_{r}^{5} u\right|^{2}\right)+\int_{Q_{T}} r^{m}\left(\rho^{3} u_{r r t t}^{2}+\rho u_{r r r r t}^{2}\right) \leq c .
$$

Proof. From (3.15), (2.3), (2.4), (2.7), (2.8), Lemmas 3.1 3.3 and Lemma 3.6, we get

$$
\begin{aligned}
\int_{I} r^{m} \rho^{2} u_{r r r t}^{2} & \leq c \int_{I} r^{m}\left(f_{t}^{2}+f_{r}^{2}+f_{r t}^{2}\right)+c \\
& \leq c .
\end{aligned}
$$

This combining (3.22) and Lemma 3.4 gives

$$
\int_{I} r^{m}\left|\partial_{r}^{5} u\right|^{2} \leq c
$$


By (2.2), (2.3), (2.4), (2.7), (2.8), Lemma 3.1, Lemma 3.3 and Lemma 3.6, we get

$$
\begin{aligned}
\int_{Q_{T}} r^{m} \rho^{3} u_{r r t t}^{2} & \leq c \int_{Q_{T}} r^{m}\left(f_{t}^{2}+f_{t t}^{2}\right)+c \\
& \leq c .
\end{aligned}
$$

Differentiating (3.15) with respect to $r$, we have

$$
\begin{aligned}
u_{r r r r t}= & \rho_{r r t} u_{t}+2 \rho_{r t} u_{r t}+\rho_{r r} u_{t t}+2 \rho_{r} u_{r t t}+\rho_{t} u_{r r t}+\rho u_{r r t t}+\left[\rho_{r t} u u_{r}+\rho_{r} u_{t} u_{r}\right. \\
& +\rho_{r} u u_{r t}+\rho_{t} u_{r}^{2}+2 \rho u_{r} u_{r t}+\rho_{t} u u_{r r}+\rho u_{t} u_{r r}+\rho u u_{r r t}+\left(\rho^{\gamma}\right)_{r r t} \\
& \left.-m r^{-1} u_{r r t}+2 m r^{-2} u_{r t}-\frac{2 m u_{t}}{r^{3}}\right]_{r}-\rho_{r r t} f-2 \rho_{r t} f_{r}-\rho_{r r} f_{t}-\rho_{t} f_{r r}(3.25) \\
& -2 \rho_{r} f_{r t}-\rho f_{r r t} .
\end{aligned}
$$

By (3.24), (3.25), (2.3), (2.4), (2.7), (2.8), Lemmas 3.13.3 and Lemma 3.5, we obtain

$$
\begin{aligned}
\int_{Q_{T}} r^{m} \rho u_{r r r r t}^{2} \leq & c \int_{Q_{T}} r^{m} \rho^{2}\left|(\sqrt{\rho})_{r}\right|^{2} u_{r t t}^{2}+c \int_{Q_{T}} r^{m} \rho^{3} u_{r r t t}^{2} \\
& +c \int_{Q_{T}} r^{m}\left(f_{r r}^{2}+f_{r t}^{2}+f_{r r t}^{2}\right)+c \\
\leq & c .
\end{aligned}
$$

The proof of Lemma 3.7 is complete.

LEMma 3.8. For any $0 \leq t \leq T$, it holds

$$
\int_{I} r^{m}\left(\left|\partial_{r}^{5} \rho\right|^{2}+\left|\partial_{r}^{5}\left(\rho^{\gamma}\right)\right|^{2}\right)+\int_{Q_{T}} r^{m}\left|\partial_{r}^{6} u\right|^{2} \leq c .
$$

Proof. Differentiating (3.16) with respect to $r$, we obtain

$$
\begin{aligned}
& \partial_{r}^{5} \rho_{t}+m r^{-1} \rho u_{r r r r r}+m r^{-1} \rho_{r} u_{r r r r}-m r^{-2} \rho u_{r r r r}+\left(4 m r^{-1} \rho_{r} u_{r r r}+6 m r^{-1} \rho_{r r} u_{r r}\right. \\
& \left.-\frac{4 m \rho u_{r r r}}{r^{2}}+\frac{12 m \rho u_{r r}}{r^{3}}-\frac{18 m \rho u_{r}}{r^{4}}+4 m r^{-1} \rho_{r r r} u_{r}\right)_{r}+m r^{-1} \rho_{r r r r r} u+m r^{-1} \rho_{r r r r} u_{r} \\
& -\frac{5 m \rho_{r r r r} u}{r^{2}}-\frac{4 m \rho_{r r r} u_{r}}{r^{2}}+\frac{8 m \rho_{r r r} u}{r^{3}}+\left[-\frac{12 m \rho_{r} u_{r r}}{r^{2}}-\frac{12 m \rho_{r r} u_{r}}{r^{2}}+\frac{24 m \rho_{r} u_{r}}{r^{3}}\right. \\
& \left.+\frac{12 m \rho_{r r} u}{r^{3}}-\frac{6 m \rho u_{r}}{r^{4}}-\frac{24 m \rho_{r} u}{r^{4}}+\frac{24 m \rho u}{r^{5}}\right]_{r}+6 \rho_{r} \partial_{r}^{5} u+\rho \partial_{r}^{6} u+15 \rho_{r r} u_{r r r r} \\
& +20 \rho_{r r r} u_{r r r}+15 \rho_{r r r r} u_{r r}+6 \partial_{r}^{5} \rho u_{r}+u \partial_{r}^{6} \rho=0 .
\end{aligned}
$$

Multiplying (3.26) by $r^{m} \partial_{r}^{5} \rho$, and using (2.3), (2.4), (2.7), (2.8), Lemma 3.1] Lemma 3.3. Lemma 3.4 and Lemma 3.7, we have

$$
\begin{aligned}
\frac{d}{d t} \int_{I} r^{m}\left|\partial_{r}^{5} \rho\right|^{2} & \leq c \int_{I} r^{m}\left|\partial_{r}^{5} \rho\right|^{2}+c \int_{I} r^{m}\left|\partial_{r}^{6} u\right|^{2}+\int_{I} r^{m}\left(\left|\partial_{r}^{5} \rho\right|^{2}\right)_{r} u+c \\
& \leq c \int_{I} r^{m}\left|\partial_{r}^{5} \rho\right|^{2}+c \int_{I} r^{m}\left|\partial_{r}^{6} u\right|^{2}+c .
\end{aligned}
$$

Similarly, we get

$$
\frac{d}{d t} \int_{I} r^{m}\left|\partial_{r}^{5}\left(\rho^{\gamma}\right)\right|^{2} \leq c \int_{I} r^{m}\left|\partial_{r}^{5}\left(\rho^{\gamma}\right)\right|^{2}+c \int_{I} r^{m}\left|\partial_{r}^{6} u\right|^{2}+c
$$


Differentiating (3.20) with respect to $r$, we get

$$
\begin{aligned}
\partial_{r}^{6} u= & \rho_{r r r r} u_{t}+4 \rho_{r r r} u_{r t}+6 \rho_{r r} u_{r r t}+4 \rho_{r} u_{r r r t}+\rho u_{r r r r t}+\left(\rho_{r} u u_{r}+\rho u_{r}^{2}+\rho u u_{r r}\right)_{r r r} \\
& +\partial_{r}^{5}\left(\rho^{\gamma}\right)+\left(-m r^{-1} u_{r r r r}+4 m r^{-2} u_{r r r}-12 m r^{-3} u_{r r}+24 m r^{-4} u_{r}-\frac{24 m u}{r^{5}}\right)_{r} \\
& -\rho_{r r r r} f-4 \rho_{r r r} f_{r}-6 \rho_{r r} f_{r r}-4 \rho_{r} f_{r r r}-\rho f_{r r r r} .
\end{aligned}
$$

(3.29), (2.3), (2.4), 2.7), (2.8), Lemma 3.1. Lemma 3.3, Lemma 3.4 and Lemma 3.7 imply

$$
\begin{aligned}
\int_{I} r^{m}\left|\partial_{r}^{6} u\right|^{2} \leq & c \int_{I} r^{m} u_{r r t}^{2}+c \int_{I} r^{m} u_{r r r t}^{2}+c \int_{I} r^{m} \rho u_{r r r r t}^{2}+c \int_{I} r^{m}\left|\partial_{r}^{5}\left(\rho^{\gamma}\right)\right|^{2} \\
& +c \int_{I} r^{m}\left(f_{r r}^{2}+f_{r r r}^{2}+f_{r r r r}^{2}\right)+c
\end{aligned}
$$

It follows from (3.27), (3.28), (3.30), (2.8), Lemma 3.3, Lemma 3.7 and Gronwall inequality that

$$
\int_{I} r^{m}\left[\left|\partial_{r}^{5} \rho\right|^{2}+\left|\partial_{r}^{5}\left(\rho^{\gamma}\right)\right|^{2}\right] \leq c .
$$

From (3.30), (3.31), (2.8), Lemma 3.3 and Lemma 3.7, we obtain

$$
\int_{Q_{T}} r^{m}\left|\partial_{r}^{6} u\right|^{2} \leq c
$$

The proof of Lemma 3.8 is complete.

It follows from (1.4) $1,(2.6),(2.7),(2.8)$ and Lemmas 3.1 3.8 that

$$
\left\|\left(\rho, \rho^{\gamma}\right)\right\|_{H_{r}^{5}}+\left\|\left(\rho_{t},\left(\rho^{\gamma}\right)_{t}\right)\right\|_{H_{r}^{4}}+\left\|\left((\sqrt{\rho})_{r},(\sqrt{\rho})_{t}\right)\right\|_{L^{\infty}\left(Q_{T}\right)} \leq c, \rho \geq \frac{\delta}{c},
$$

and

$$
\begin{aligned}
& \int_{I} r^{m}\left(\rho^{3} u_{t t}^{2}+\rho^{4} u_{r t t}^{2}+r^{-2} \rho^{4} u_{t t}^{2}+\rho^{2} u_{r r r t}^{2}+\rho u_{r r t}^{2}+u_{r t}^{2}+r^{-2} u_{t}^{2}+\left|\partial_{r}^{5} u\right|^{2}+\left|\partial_{r}^{4} u\right|^{2}\right. \\
& \left.+u_{r r r}^{2}+u_{r r}^{2}+u_{r}^{2}+r^{-2} u^{2}\right)+\int_{Q_{T}} r^{m}\left(\rho u_{t t}^{2}+\rho^{2} u_{r t t}^{2}+\rho^{5} u_{t t t}^{2}+\rho^{3} u_{r r t t}^{2}+\rho u_{r r r t}^{2}\right. \\
& \left.+u_{r r t}^{2}+u_{r r r t}^{2}+\left|\partial_{r}^{6} u\right|^{2}\right) \leq c .
\end{aligned}
$$

By (3.32) and (3.33), we complete the proof of Theorem 3.1 .

\section{Proof of Theorem 1.2}

Since (3.32) and (3.33) are uniform for $b$ and $\delta$, it suffices to prove Theorem 1.2 for the case $b<\infty$. We follow the strategy as the proof of Theorem 1.1 and use Theorem 3.1. After taking $\delta \rightarrow 0$ (take subsequence if necessary), we get a solution $(\rho, u)$ of (1.4)-(1.6) satisfying

$$
\left\{\begin{array}{l}
\left(\rho, \rho^{\gamma}\right) \in L^{\infty}\left([0, T] ; H^{5}(I)\right),\left((\sqrt{\rho})_{r},(\sqrt{\rho})_{t}\right) \in L^{\infty}\left(Q_{T}\right) \\
\left(\rho_{t},\left(\rho^{\gamma}\right)_{t}\right) \in L^{\infty}\left([0, T] ; H^{4}(I)\right), u \in L^{\infty}\left([0, T] ; H^{5}(I)\right) \cap L^{2}\left([0, T] ; H^{6}(I)\right), \\
u_{t} \in L^{\infty}\left([0, T] ; H_{0}^{1}(I)\right) \cap L^{2}\left([0, T] ; H^{3}(I)\right) \\
\left(\sqrt{\rho} \partial_{r}^{2} u_{t}, \rho \partial_{r}^{3} u_{t}\right) \in L^{\infty}\left([0, T] ; L^{2}(I)\right) \cap L^{2}\left([0, T] ; H^{1}(I)\right) .
\end{array}\right.
$$


It follows from $u \in L^{\infty}\left([0, T] ; H^{5}(I)\right) \cap L^{2}\left([0, T] ; H^{6}(I)\right), u_{t} \in L^{2}\left([0, T] ; H^{3}(I)\right)$, (1.4) 1 , (2.6) and the similar arguments as [3, 4] that

$$
\rho, \rho^{\gamma} \in C\left([0, T] ; H^{5}(I)\right) .
$$

Denote $\rho(\mathbf{x}, t)=\rho(r, t), \mathbf{u}(\mathbf{x}, t)=u(r, t) \frac{\mathbf{x}}{r}$, then $(\rho, \mathbf{u})$ is the unique solution to (1.1)-(1.3) with the regularities in Theorem[1.2, The proof of Theorem 1.2 is complete.

REMARK 3.1. By our method, it seems that the regularities of $\mathbf{u}$ could not be improved to $L^{\infty}\left([0, T] ; D^{6}(\Omega)\right)$ and $L^{2}\left([0, T] ; D^{8}(\Omega)\right)$, even if the initial data, $\mathbf{f}$ and $\mathrm{g}_{1}$ of (1.1)-(1.3) are smooth enough. More precisely, based on (3.32) and (3.33), by using similar arguments as in the proofs of Theorem 1.1 and 1.2, we get the next two a priori estimates about $u$ for (1.4)-(1.6).

$$
\int_{I} r^{m} \rho^{7} u_{t t t}^{2}+\int_{Q_{T}} r^{m} \rho^{6}\left(u_{r t t t}^{2}+r^{-2} u_{t t t}^{2}\right) \leq c
$$

and

$$
\int_{I} r^{m} \rho^{8}\left(u_{r t t t}^{2}+r^{-2} u_{t t t}^{2}\right)+\int_{Q_{T}} r^{m} \rho^{9} u_{t t t t}^{2} \leq c .
$$

(3.35) and (3.36) respectively implies

$$
\int_{I} r^{m} \rho\left|\partial_{r}^{6} u\right|^{2}+\int_{Q_{T}} r^{m}\left|\partial_{r}^{7} u\right|^{2} \leq c
$$

and

$$
\int_{I} r^{m} \rho^{2}\left|\partial_{r}^{7} u\right|^{2}+\int_{Q_{T}} r^{m} \rho\left|\partial_{r}^{8} u\right|^{2} \leq c
$$

But the appearance of vacuum stops us from obtaining the regularities of $\mathbf{u}$ in $L^{\infty}\left([0, T] ; D^{6}(\Omega)\right)$ and $L^{2}\left([0, T] ; D^{8}(\Omega)\right)$ from (3.37) and (3.38). Is there another way to get further regularities of the solutions, such as $L^{\infty}\left([0, T] ; D^{6}(\Omega)\right)$-regularity? This is an interesting problem.

\section{Acknowledgment.}

The authors would like to thank the anonymous referees for their constructive suggestions and kindly comments. S.J. Ding was supported by the National Basic Research Program of China (973 Program) (No.2011CB808002), the National Natural Science Foundation of China (No.11071086), and the University Special Research Foundation for Ph.D Program (No.20104407110002). L. Yao was supported by the National Natural Science Foundation of China \#11101331 and China Postdoctoral Science Foundation funded project \#20100481359, \#201104676. C.J. Zhu was supported by the National Natural Science Foundation of China \#10625105, \#11071093, the PhD specialized grant of the Ministry of Education of China \#20100144110001, and the Special Fund for Basic Scientific Research of Central Colleges \#CCNU10C01001. 
[1] H. Beirão da Veiga, Long time behavior for one-dimensional motion of a general barotropic viscous fluid. Arch. Rational Mech. Anal., 108(1989), 141-160.

[2] H.J. Choe, H. Kim, Strong solutions of the Navier-Stokes equations for isentropic compressible fluids. J. Differential Equations, 190(2003), 504-523.

[3] Y. Cho, H.J. Choe, H. Kim, Unique solvability of the initial boundary value problems for compressible viscous fluids. J. Math. Pures Appl., 83(2004), 243-275.

[4] H.J. Choe, H. Kim, Global existence of the radially symmetric solutions of the Navier-Stokes equations for the isentropic compressible fluids. Math. Methods Appl. Sci., 28(2005), 1-28.

[5] Y. Cho, H. Kim, On classical solutions of the compressible Navier-Stokes equations with nonnegative initial densities. Manuscripta Math., 120(2006), 91-129.

[6] S.J. Ding, H.Y. Wen, C.J. Zhu, Global classical large solutions of $1 D$ compressible NavierStokes equations with density-dependent viscosity and vacuum. J. Differential Equations, 251(2011), 1696-1725.

[7] L.C. Evans, Partial Differential Equations, Graduate Studies in Mathematics, Vol. 19, 1998.

[8] J.S. Fan, S. Jiang, G.X. Ni, Uniform boundedness of the radially symmetric solutions of the Navier-Stokes equations for isentropic compressible fluids. Osaka J. Math., 46(2009), 863876.

[9] E. Feireisl, A. Novotný, H. Petzeltová, On the existence of globally defined weak solutions to the Navier-Stokes equations. J. Math. Fluid Mech., 3(2001), 358-392.

[10] G.P. Galdi, An Introduction to the Mathematical Theory of the Navier-Stokes Equations, Springer-Verlag, New York, 1994.

[11] D. Hoff, Spherically symmetric solutions of the Navier-Stokes equations for compressible, isothermal flow with large, discontinuous initial data. Indiana Univ. Math. J., 41(1992), 1225-1302.

[12] S. Jiang, Global spherically symmetric solutions to the equations of a viscous polytropic ideal gas in an exterior domain. Comm. Math. Phys., 178(1996), 339-374.

[13] S. Jiang, P. Zhang, On spherically symmetric solutions of the compressible isentropic NavierStokes equations. Comm. Math. Phys., 215(2001), 559-581.

[14] B. Kawohl, Global existence of large solutions to initial-boundary value problems for a viscous, heat-conducting, one-dimensional real gas. J. Differential Equations, 58(1985), 76-103.

[15] A.V. Kazhikhov, Stabilization of solutions of an initial-boundary-value problem for the equations of motion of a barotropic viscous fluid. Differ. Equ., 15(1979), 463-467.

[16] P.L. Lions, Mathematical Topics in Fluid Dynamics, Vol. 2, Compressible Models, Oxford Science Publication, Oxford, 1998.

[17] A. Matsumura, T. Nishida, The initial value problem for the equations of motion of viscous and heat-conductive gases. J. Math. Kyoto Univ., 20(1980), 67-104.

[18] A. Matsumura, T. Nishida, Initial-boundary value problems for the equations of motion of compressible viscous and heat-conductive fluids. Comm. Math. Phys., 89(1983), 445-464.

[19] R. Salvi, I. Straškraba. Global existence for viscous compressible fluids and their behavior as $t \rightarrow \infty$. J. Fac. Sci. Univ. Tokyo Sect. IA Math., 40(1993), 17-51.

[20] A. Tani, On the first initial-boundary value problem of compressible viscous fluid motion. Publ. Res. Inst. Math. Sci. Kyoto Univ., 13(1977), 193-253.

[21] V.A. Weigant, Example of non-existence in the large for the problem of the existence of solutions of Navier-Stokes equations for compressible viscous barotropic fluids. Dokl. Akad. Nauk., 339(1994), 155-156, in Russian.

[22] Z.P. Xin, Blowup of smooth solutions to the compressible Navier-Stokes equation with compact density. Comm. Pure Appl. Math., 51(1998), 229-240. 